\title{
Simultaneous observation of the electron acceleration and ion deceleration over lunar magnetic anomalies
}

\author{
Yoshifumi Saito $^{1}$, Masaki N. Nishino ${ }^{1}$, Masaki Fujimoto ${ }^{1}$, Tadateru Yamamoto ${ }^{1}$, Shoichiro Yokota ${ }^{1}$, Hideo Tsunakawa $^{2}$, \\ Hidetoshi Shibuya ${ }^{3}$, Masaki Matsushima ${ }^{2}$, Hisayoshi Shimizu ${ }^{4}$, and Futoshi Takahashi ${ }^{2}$ \\ ${ }^{1}$ Institute of Space and Astronautical Science, Japan Aerospace Exploration Agency, \\ 3-1-1 Yoshinodai, Chuo, Sagamihara, Kanagawa 252-5210, Japan \\ ${ }^{2}$ Tokyo Institute of Technology, 2-12-1 Ookayama, Meguro-ku, Tokyo 152-8551, Japan \\ ${ }^{3}$ Kumamoto University, 2-39-1, Kurokami, Kumamoto 860-8555, Japan \\ ${ }^{4}$ Earthquake Research Institute, University of Tokyo, 1-1-1 Yayoi, Bunkyo-ku, Tokyo 113-0032, Japan
}

(Received March 14, 2011; Revised July 4, 2011; Accepted July 19, 2011; Online published March 8, 2012)

\begin{abstract}
At $\sim 25 \mathrm{~km}$ altitude over magnetic anomalies on the Moon, the deceleration of the solar wind ions, acceleration of the solar wind electrons parallel to the magnetic field, and heating of the ions reflected by magnetic anomalies were simultaneously observed by MAP-PACE on Kaguya. Deceleration of the solar wind ions was observed for two major solar wind ion compositions: protons and alpha particles. Deceleration of the solar wind had the same $\Delta E / q(\Delta E$ : deceleration energy, $q$ : charge) for both protons and alpha particles. In addition, the acceleration energy of the electrons was almost the same as the deceleration energy of the ions. This indicates the existence of an anti-moonward electric field over the magnetic anomaly above the altitude of Kaguya. The reflected ions were observed in a much larger area than the area where magnetic field enhancement was observed. These reflected ions had a higher temperature and lower bulk velocity than the incident solar wind ions. This suggests the existence of a non-adiabatic dissipative interaction between solar wind ions and lunar magnetic anomalies below Kaguya.
\end{abstract}

Key words: Moon, plasma, magnetic anomaly, solar wind.

\section{Introduction}

It is well-known that the Moon has neither a global intrinsic magnetic field nor a thick atmosphere. Unlike the case of the Earth, where the intrinsic global magnetic field prevents the solar wind from penetrating into the magnetosphere, the solar wind directly impacts the lunar surface. Since the discovery of the lunar crustal magnetic field in the 1970s (Sharp et al., 1973; Fuller, 1974; Howe et al., 1974; Anderson et al., 1975), several studies concerning the interaction between the solar wind and the lunar magnetic anomalies have been made, including numerical simulations (Harnett and Winglee, 2000, 2002, 2003) and observations by lunar orbiters. The interaction between the solar wind and lunar magnetic anomalies was first detected by Explorer 35 as limb shocks (Ness et al., 1968; Colburn, 1971; Criswell, 1973). Using Apollo 15 and 16 subsatellite data, Russell and Lichtenstein (1975) suggested that the limb compressions are due to the deflection of the solar wind by the lunar surface field near the lunar terminators. MAG/ER on Lunar Prospector found heating of the solar wind electrons presumably due to the interaction between the solar wind and the lunar magnetic anomalies and the existence of the mini-magnetosphere was suggested (Lin et al., 1998). Using Lunar Prospector MAG data, Kurata et al.

Copyright (C) The Society of Geomagnetism and Earth, Planetary and Space Sciences (SGEPSS); The Seismological Society of Japan; The Volcanological Society of Japan; The Geodetic Society of Japan; The Japanese Society for Planetary Sciences; TERRAPUB.

doi:10.5047/eps.2011.07.011
(2005) found a magnetic field signature indicating the presence of a mini-magnetosphere above the Reiner Gamma magnetic anomaly region in the solar wind. Halekas et al. (2006, 2008a) found that limb shocks are clearly associated with lunar crustal sources by analyzing Lunar Prospector data. Observation of the possible inner region of a lunar mini-magnetosphere was reported by Halekas et al. (2008b). Recently, Wieser et al. (2010) detected minimagnetospheres on the lunar surface using energetic neutral atom imager on Chandrayaan-1. Saito et al. (2010) also found mini-magnetospheres by detecting the deficiency of backscattered solar wind protons at low altitude of $\sim 50 \mathrm{~km}$ using low energy ion data obtained by Kaguya. Solar wind ion reflection by lunar magnetic anomalies was reported by Kaguya (Saito et al., 2010) and Chandrayaan-1 (Lue et al., 2011). Saito et al. (2010) found that more than $10 \%$ of the incident solar wind ions were reflected by lunar magnetic anomalies. Lue et al. (2011) found an average reflection efficiency of $\sim 10 \%$ and a maximum reflection efficiency of $\sim 50 \%$ for the strongest magnetic anomalies. As a reflection mechanism, Lue et al. (2011) suggested the existence of charge separation. Halekas et al. (2010) also mentioned the existence of charge separation around the magnetic anomalies. Despite all the efforts to understand the interaction between the solar wind and lunar magnetic anomalies, the details of the interaction are still unclear. In this paper, the first simultaneous observation of low energy electrons and ions over lunar magnetic anomalies are presented in order to understand the plasma structure over mini-magnetospheres. 


\section{Instrumentation}

MAgnetic field and Plasma experiment-Plasma energy Angle and Composition Experiment (MAP-PACE) on Kaguya (Saito et al., 2008a, 2010) completed its $\sim 1.5$ year observation of low energy charged particles around the Moon. Kaguya (SELENE) was launched on 14 September 2007 by an H2A launch vehicle from Tanegashima Space Center in Japan (Kato et al., 2010). Kaguya was inserted into a circular lunar polar orbit of $100 \mathrm{~km}$ altitude and continued observation for nearly 1.5 years until it impacted the Moon on 10 June 2009. During the last 5 months, the orbit was lowered to $\sim 50 \mathrm{~km}$ altitude between January 2009 and April 2009, and some orbits had a lower perilune altitude of $\sim 10 \mathrm{~km}$ after April 2009.

MAP-PACE consisted of four sensors: ESA (Electron Spectrum Analyzer)-S1, ESA-S2, IMA (Ion Mass Analyzer), and IEA (Ion Energy Analyzer). Since each sensor had a hemispherical field of view, two electron sensors and two ion sensors which were installed on the spacecraft panels opposite each other could cover the full 3-dimensional phase space of low energy electrons and ions. One of the ion sensors, IMA, was an energy mass spectrometer that measured mass identified ion energy spectra (Tanaka et al., 2009; Yokota et al., 2009).

The Lunar MAGnetometer (MAP-LMAG) was another component that constituted MAP. MAP-LMAG was a triaxial flux gate magnetometer that was equipped at the top plate of a $12 \mathrm{~m}$ long mast to reduce an offset of the interference magnetic field caused by the spacecraft (Shimizu et al., 2008; Takahashi et al., 2009; Matsushima et al., 2010; Tsunakawa et al., 2010). LMAG measured the vector magnetic field with a sampling frequency of $32 \mathrm{~Hz}$ and a resolution of $0.1 \mathrm{nT}$.

\section{Observation}

Figure 1 shows an example of the electrons and ions over magnetic anomalies observed at $\sim 100 \mathrm{~km}$ altitude. The data were obtained between 0710 UT and 0910 UT on 29 May 2008, when the Moon was in the solar wind as shown in Fig. 1(a). The orbital plane of Kaguya was about 55 deg. apart from the noon-midnight plane. Figure 1(c) and (d) show electron $E$ - $t$ spectrograms obtained by ESA-S2 (anti-moon looking electron analyzer) and ESA-S1 (moon looking electron analyzer), respectively. Figure 1(e) is an $E$ - $t$ spectrogram of the solar wind ions observed by IEA (anti-moon looking ion analyzer). Alpha particles as well as protons were observed between 0740 UT and 0840 UT. Figure 1(f) is an $E$ - $t$ spectrogram of the ions observed by IMA (moon looking ion analyzer). Since IMA's hemispherical field of view always faces the Moon, it observes solar wind ions briefly before entering and briefly after exiting the lunar wake as shown in Fig. 1(b) (satellite positions (2) and (3)). The intense ions observed by IMA at $\sim 1 \mathrm{keV} / \mathrm{q}$ between 0710 UT and 0745 UT and between 0835 UT and 0900 UT were solar wind ions. The tenuous ions observed between 0750 UT and 0815 UT with energy slightly lower than the solar wind proton energy were solar wind protons backscattered at the lunar surface (Saito et al., 2008b). The ions observed between 0745 UT and 0800 UT with lower energy than the backscattered protons were ions originat- ing from the lunar surface/lunar exosphere (Yokota et al., 2009). The ions observed between 0750 UT and 0810 UT with higher energy than the solar wind protons were ions pick-up accelerated by the solar wind convection electric field (Saito et al., 2008b). The intense ions observed between 0815 UT and 0835 UT (indicated by a blue box) were the ions reflected by magnetic anomalies on the lunar surface. When magnetically reflected ions were observed, Kaguya was at $-5 \mathrm{deg}$. $\sim-70 \mathrm{deg}$. Latitude, $-169.0 \mathrm{deg}$. Longitude over the South Pole Aitken region. Enhancement of the magnetic field intensity was observed (see Fig. 1(g)) when magnetically reflected ions were observed. This enhancement was caused by magnetic anomalies on the lunar surface since the solar wind IMF measured by ACE (propagation time corrected; see Fig. 1(i)) showed no enhancement. The flux of the magnetically reflected solar wind ions was much higher than that of the backscattered solar wind protons. When magnetically reflected ions were observed, the electrons were often heated and the incident solar wind ions were sometimes slightly decelerated.

Figure 2 compares the TOF (Time Of Flight) profile of the three different ion populations: solar wind ions, magnetically reflected ions, and ions backscattered at the lunar surface. Figure 2(c), (d), and (e) show Energy/charge-TOF spectrograms below the time of flight of $100 \mathrm{nsec}$. Since IMA is an LEF (Linear Electric Field) TOF mass spectrometer (Yokota et al., 2005), incident ions are converted to positively charged particles, negatively charged particles or neutral particles when they pass through the thin carbon foil inside the analyzer. The charge state of the detected (after passing through the carbon foil) particles is also indicated in the figure. Figure 2(f), (g), and (h) show energy integrated TOF profiles. Figure 2(i), (j) and (k) focus on the low count part of the energy integrated TOF profiles (indicated by a blue box in Fig. 2(f), (g), and (h)) in order to show the alpha particles clearly. The mass profiles of the solar wind (Fig. 2(e), (h), and (k)) show both protons and alpha particles as major and second major components. On the other hand, the mass profiles of the ions backscattered at the lunar surface (Fig. 2(c), (f), and (i)) show only protons as already reported by Saito et al. (2008b). It is expected that alpha particles as well as protons are observed as magnetically reflected ions if the magnetic mirror reflection is ideal. Indeed alpha particles were observed as shown in Fig. 2(d). However, the energy of the alpha particles is lower than that of the alpha particles in the solar wind shown in Fig. 2(e). In addition, the simultaneously measured magnetically reflected protons show a much higher temperature than that of the incident solar wind protons.

Figure 3 shows an example of the magnetically reflected solar wind ions (indicated by a blue box) observed at $\sim 25 \mathrm{~km}$ altitude. The format of the figure is the same as Fig. 1. The signature of a magnetic anomaly was observed by magnetometer at 0750 UT when Kaguya was at -26.4 deg. Latitude, 177.7 deg. Longitude over the South Pole Aitken region (see Fig. 3(g)). At $\sim 25 \mathrm{~km}$ altitude, the interaction between the solar wind ions and the lunar magnetic anomalies was remarkable, with a clear deceleration of the incident solar wind ions (see Fig. 3(e)) and heating of the reflected ions (see Fig. 3(f)) as well as acceleration of the 


\section{KAGUYA MAP-PACE 20080529071000 - 091000 \\ (a) \\ (b) Field Of View of MAP-PACE sensors}

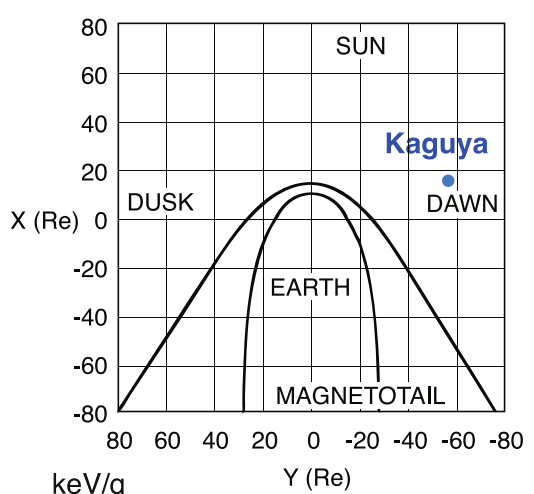

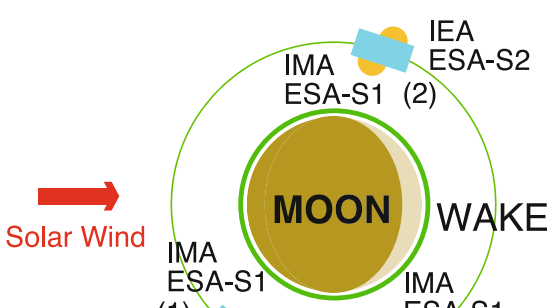

(1)

IEA ESA-S2
(3) ESA-S1

IEA

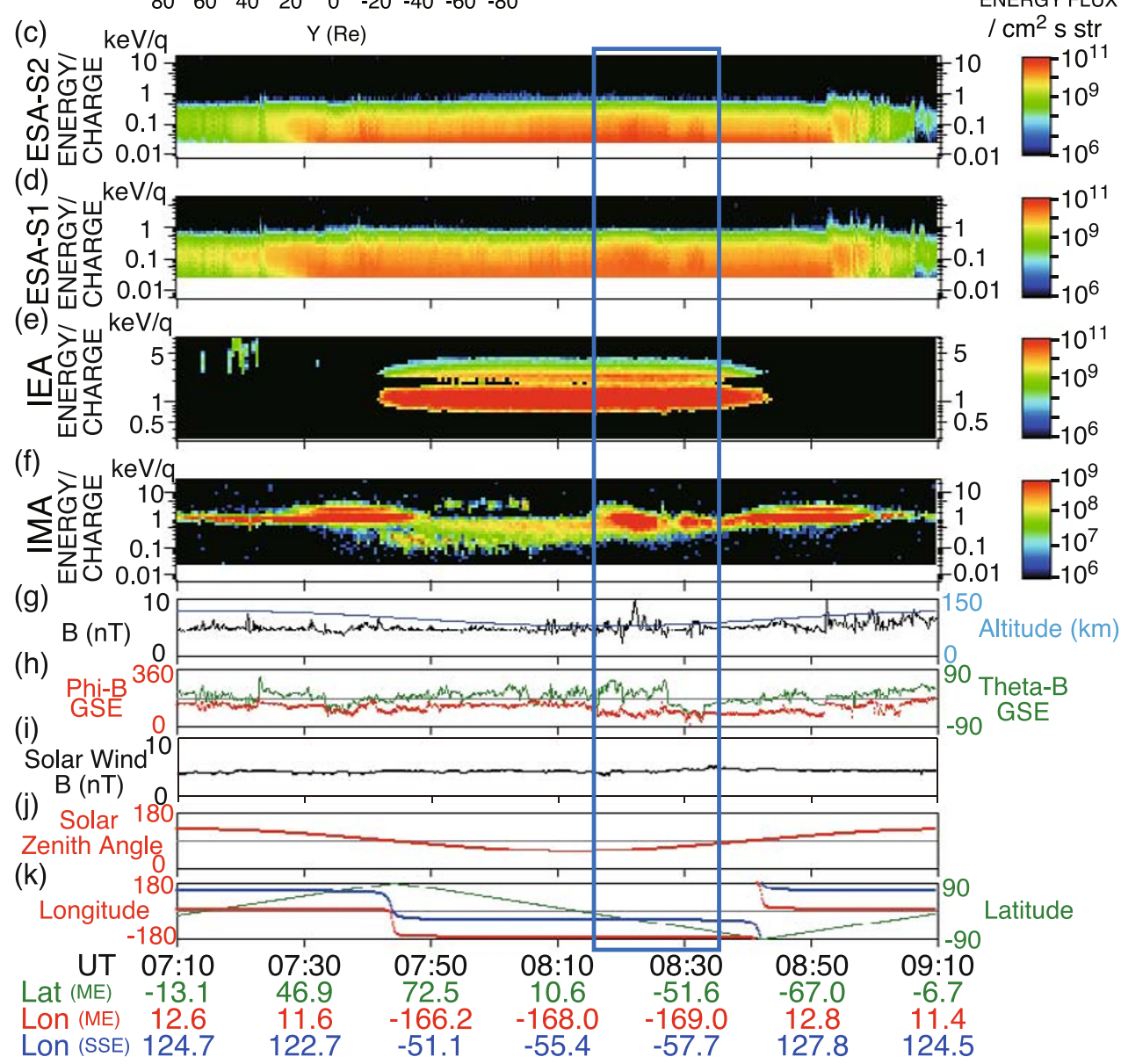

Fig. 1. Electrons and ions over magnetic anomalies observed at $\sim 100 \mathrm{~km}$ altitude. Magnetically reflected solar wind ions were observed by IMA when Kaguya flew over magnetic anomalies during the time period indicated by a blue box. (a) Position of the Moon in the GSE coordinate system. Nominal magnetopause and bow shock boundary positions are also shown. (b) Field of view configuration of MAP-PACE sensors. Magnetically reflected ions were observed at satellite positions. (1), (2), and (3) indicate the positions where Moon-facing sensors observed solar wind. (c)-(f) Omni-directional $E$ - $t$ spectrograms from the PACE sensors. (g) Magnetic field intensity and altitude of Kaguya. (h) Magnetic field direction in GSE polar coordinates. (i) Solar wind IMF measured by ACE shifted by 50 minutes taking into account the propagation time. (j) Solar zenith angle of Kaguya. (k) Latitudinal/longitudinal position of Kaguya in the Mean Earth/Polar Axis (ME) coordinate system and the Selenocentric Soar Ecliptic (SSE) coordinate system.

electrons (see Fig. 3(c) and (d)). It is clear that the deceleration of the solar wind ions was observed for both two major solar wind ion compositions: protons and alpha particles. It is also clear that the reflected ions were observed over a much larger area than the area where a strong magnetic field and solar wind ion deceleration were observed. Before encountering the magnetic anomaly, the solar wind bulk flow direction was nearly parallel to the magnetic field.
Over the magnetic anomaly, the angle between the solar wind bulk flow and the magnetic field became $60^{\circ} \sim 90^{\circ}$, which shows a solar wind magnetic field pile-up over the magnetic anomaly.

Figure 4 shows $E$ - $t$ spectrograms of electrons (Fig. 4(b)) and ions (Fig. 4(c)) focusing on the period when ion deceleration and electron acceleration were observed. Figure 4(a) shows the pitch angle distribution of electrons 


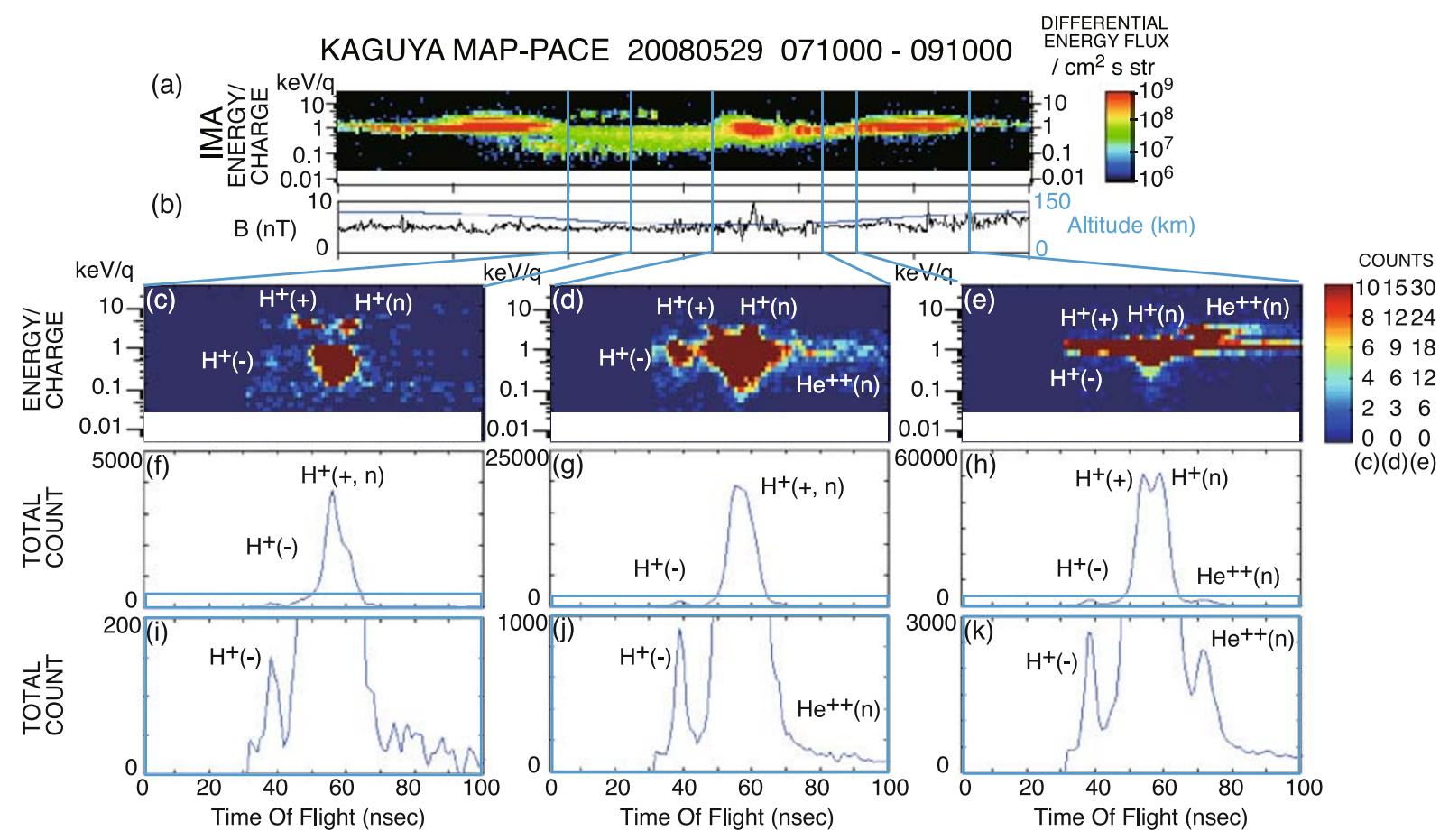

Fig. 2. Time Of Flight (TOF) profiles of the solar wind ions, magnetically reflected ions, and ions backscattered at the lunar surface. (a) $E$ - $t$ spectrogram of the ions from the Moon observed by IMA. (b) Magnetic field intensity and altitude of Kaguya. (c)-(e) Energy/charge-TOF spectrograms below the time of flight of $100 \mathrm{nsec}$. (f)-(h) Energy integrated TOF profile. (i)-(k) Low count part of the energy integrated TOF profile. Charge state of the detected particles is indicated in parentheses in panels (c)-(k).

integrated over the observed energy range. The pitch angle $0 \mathrm{deg}$. is the parallel direction toward the Moon. The accelerated electrons were heated and had mostly parallel velocity toward the Moon surface. The deceleration of the ions was observed simultaneously with the electron acceleration and the deceleration was clearly observed for both protons and alpha particles. In order to investigate the energy relation of the deceleration/acceleration of the ions/electrons in detail, energy spectra of the electrons/ions before and after acceleration/deceleration are compared. The selected period is indicated by blue and orange arrows in Fig. 4(b, c).

Figure 5(a) shows electron energy spectra before (blue line) and after (orange line) the acceleration. Comparing them, we can see that the electrons are accelerated by $\sim+150$ eV/q. In Fig. 5(c), the number flux of electrons before acceleration is shifted by $+150 \mathrm{eV} / \mathrm{q}$. The shifted line corresponds well with the number flux of electrons after acceleration. We can also see that the accelerated electrons are heated, compared with the electrons before acceleration. Figure 5(b) shows the ion energy spectra before (blue line) and after (orange line) the deceleration. Since the energy spectra have two peaks corresponding to protons and alpha particles, the deceleration of protons and alpha particles can be calculated separately. We can see that both protons and alpha particles are decelerated by $\sim 150 \mathrm{eV} / \mathrm{q}$. In Fig. 5(d), the number flux of ions before deceleration is shifted by $-150 \mathrm{eV} / \mathrm{q}$. The shifted line corresponds well with the number flux of ions after deceleration. Simultaneously with the deceleration, protons and alpha particles were slightly heated since the separation between the two peaks became less clear. The deceleration of the solar wind had the same $\Delta E / q(\Delta E:$ deceleration energy, $q$ : charge) for protons and alpha particles, and it was nearly the same as the acceleration of the solar wind electrons $\Delta E$. This indicates the existence of a static electric field over the dayside magnetic anomaly above the Kaguya altitude.

Figure 6 shows the velocity moments of electrons and ions during the same time interval as shown in Fig. 3. The density of ions from the Moon observed by IMA is shown by a red line while the solar wind ion density observed by IEA and the electron density observed by ESA are shown by blue and green lines in Fig. 6(a). The density enhancement of the ions from the Moon observed between 0700 UT and 0720 UT and between 0805 UT and 0815 UT were signatures of solar wind ions since the moon-looking ion analyzer IMA observed the solar wind before and after staying in the lunar wake. A density enhancement caused by magnetically reflected ions was observed around 0750 UT. We can see that the density of the magnetically reflected ions sometimes reaches as high as $\sim 50 \%$ of the incident solar wind ion density. Figure 6(b)-(d) focuses on the time period when Kaguya flew over a magnetic anomaly. Enhancement of the magnetic field intensity can be seen in Fig. 6(b). We can see that reflected ions were observed over a much larger area than the magnetic anomaly region (see Fig. 6(c)). Figure 6(d) compares the incident solar wind bulk velocity and the bulk velocity of the reflected ions. It is clear that the reflected ions had a lower bulk velocity than the incident solar wind ions. The reflected ion temperature was higher than the incident solar wind ions as we can see in Fig. 6(b). This suggests that some heating mechanism of ions existed at the lower altitude than Kaguya. The temperature of the electrons was also enhanced when reflected ions were observed. 
KAGUYA MAP-PACE $20090423064000-084000$

(a)

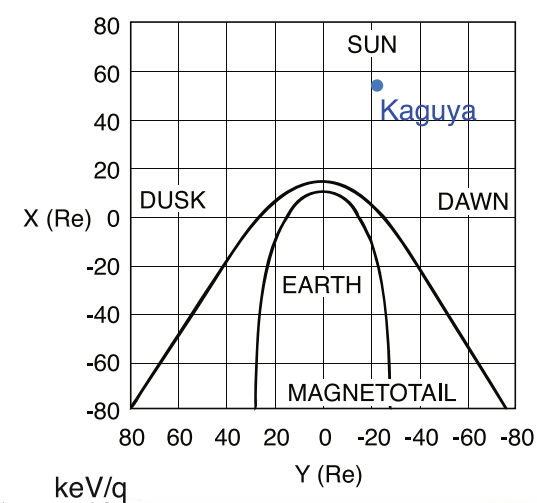

(b) Field Of View of MAP-PACE sensors
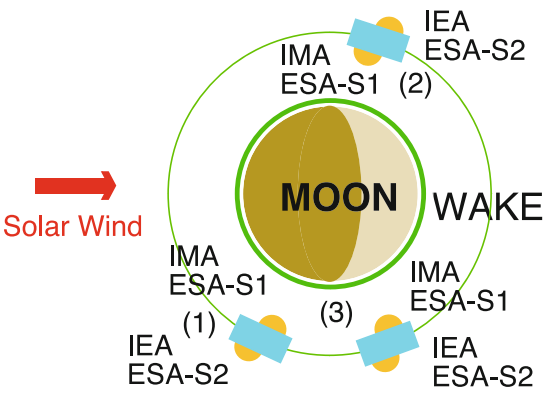

SA-S2

DIFFERENTIAL ENERGY FLUX

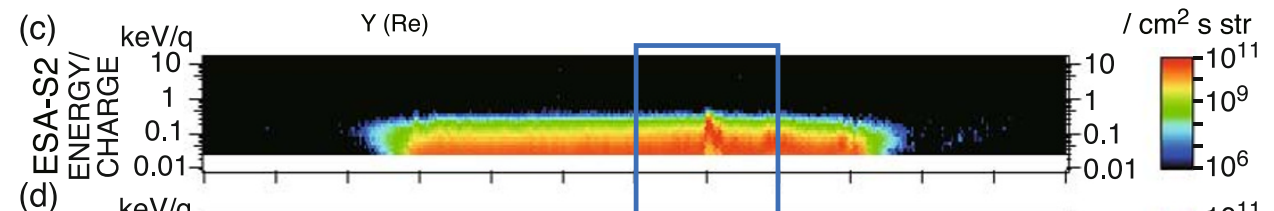

\section{(d)}

c)

(e)

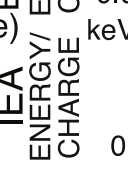

\section{(f) \\ 这岕}

0.5

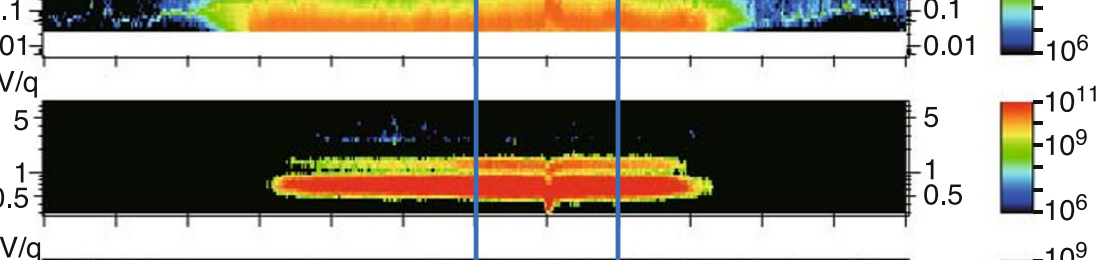

\section{(g)} 넌 0.01

0.0

(h)

(i)

GSi-B 36

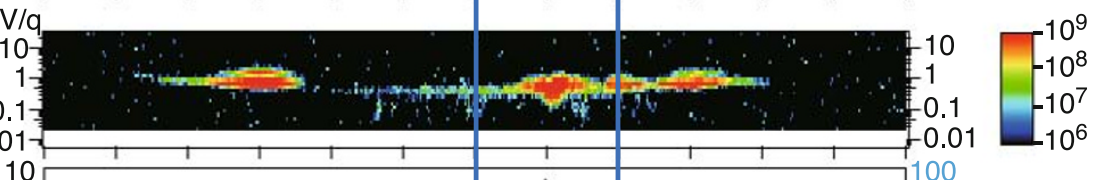

Solar Wind

Zenith Angle

(k)

Longitude

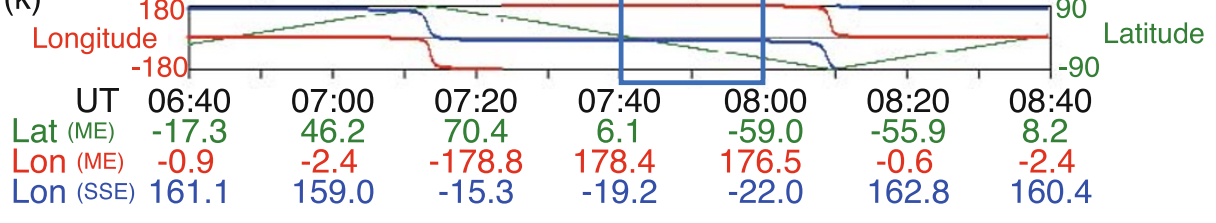

Fig. 3. Electrons and ions over magnetic anomalies observed at $\sim 25 \mathrm{~km}$ altitude. Format is the same as Fig. 1. The propagation time of the solar wind IMF measured by ACE shown in Panel (i) is 43 minutes. Magnetically reflected solar wind ions were observed by IMA when Kaguya flew over magnetic anomalies during the time period indicated by a blue box.

\section{Discussion}

The data obtained by MAP-PACE at $\sim 25 \mathrm{~km}$ altitude over lunar magnetic anomalies clearly showed acceleration of the electrons and deceleration of the ions. In terms of the "mini-magnetosphere," the region where electron acceleration and ion deceleration were observed was above (outside) the "mini-magnetosphere" since the incident solar wind still had a high density and high velocity toward the Moon. Deceleration of the solar wind had the same $\Delta E / q$ for protons and alpha particles, and it was nearly the same as the acceleration of the solar wind electrons $\Delta E$. This indicates the existence of a static anti-moonward electric field over the magnetic anomaly above Kaguya at $\sim 25 \mathrm{~km}$ altitude. The existence of an anti-moonward electric field over the magnetic anomalies was suggested by Halekas et al. (2010) and Lue et al. (2011) based on the Lunar Prospector low energy electron measurements and the Chandrayaan-1 low energy ion measurements, respectively. We have proved the existence of a static anti-moonward electric field by simultaneous measurements of low energy electrons and ions. Simultaneously with the decelerated incident solar wind ions, a significant amount of reflected ions were observed. The reflected ions were observed over a much larger area than the magnetic anomaly region. Since 
20090423 MAP-PACE IEA ESA S1 \& S2 MAP-LMAG

(a)

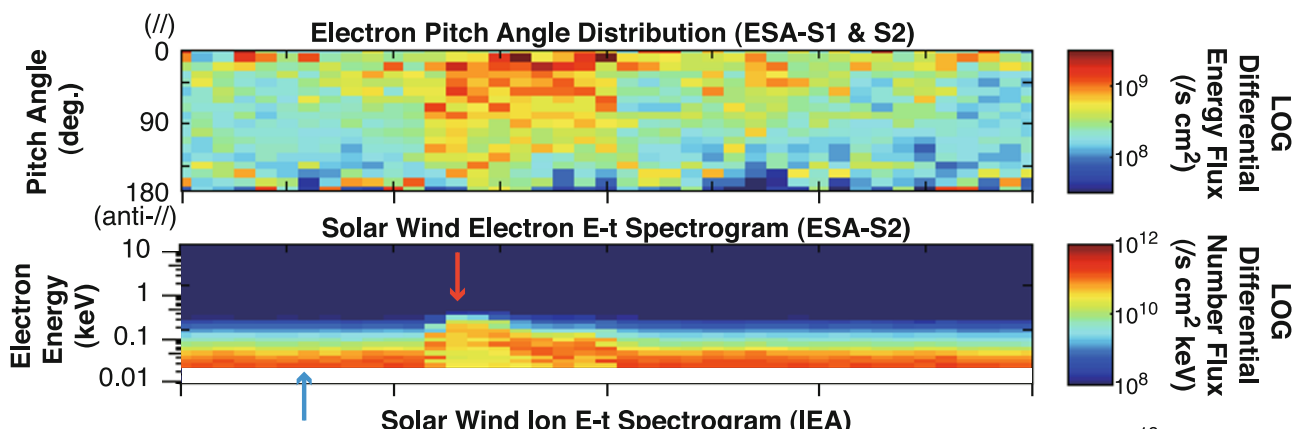

(c)

(b)

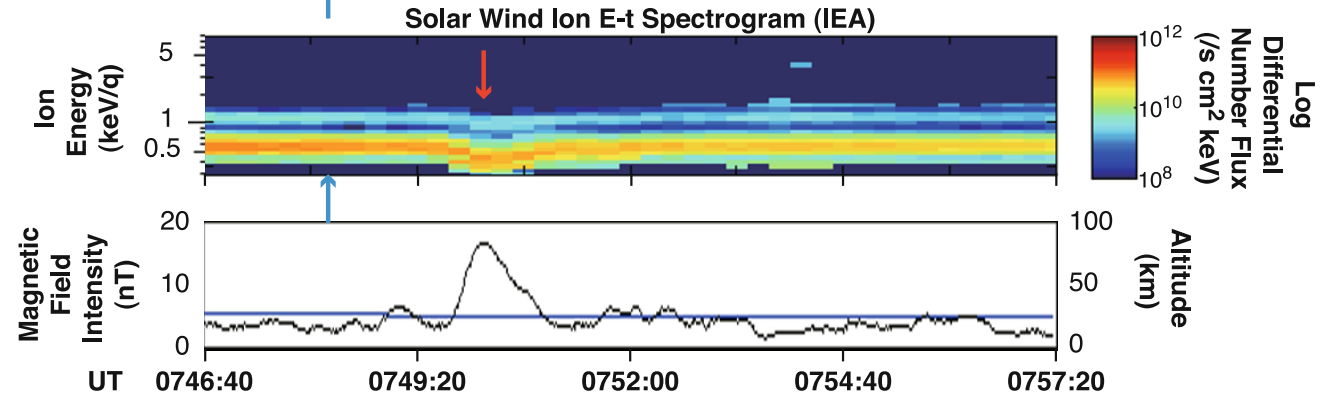

Fig. 4. Electron acceleration and ion deceleration observed over a magnetic anomaly. (a) Pitch angle distribution of electrons. Pitch angle 0 deg. is the parallel direction toward the Moon. (b) (c) $E-t$ spectrograms of electrons and ions in the period when ion deceleration and electron acceleration were observed. Blue and orange arrows show before and after acceleration/deceleration, respectively. (d) Magnetic field intensity and the altitude of Kaguya.

20090423 MAP-PACE ESA-S2 \& IMA

(a)

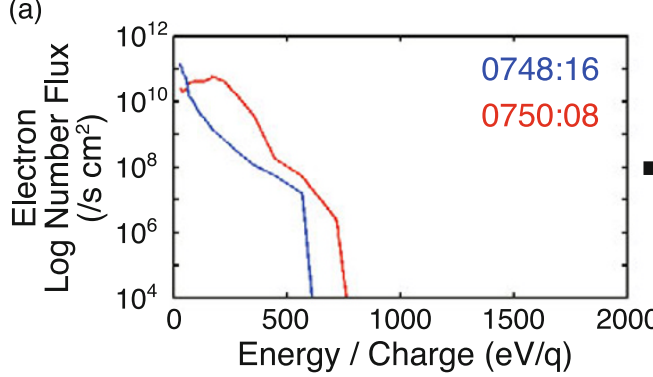

(c)

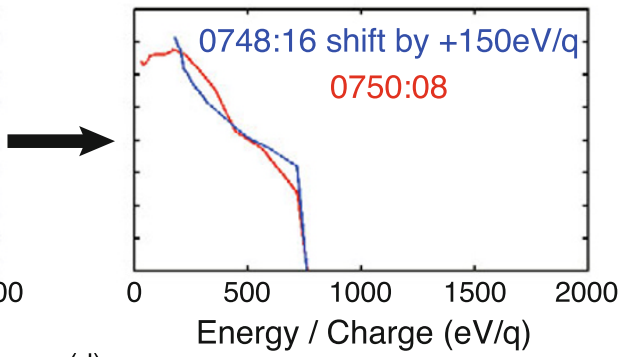

(b)

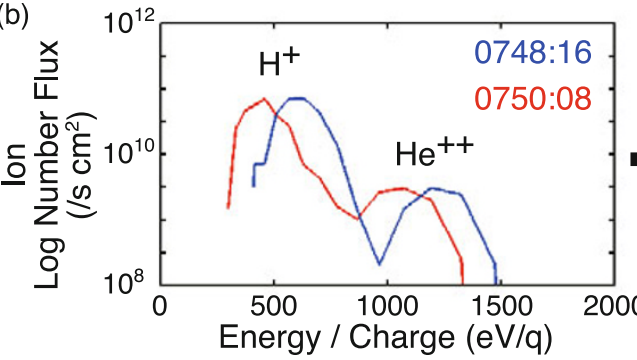

(d)

Energy / Charge (eV/q)

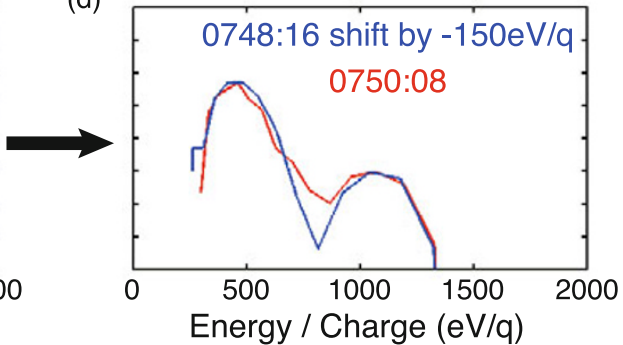

Fig. 5. Energy spectra of the electrons/ions before and after acceleration/deceleration. (a) Number flux of electrons before (0748:16 UT) and after (0750:08 UT) acceleration. (b) Number flux of ions before (0748:16 UT) and after (0750:08 UT) deceleration. (c) Number flux of electrons before acceleration is shifted by $+150 \mathrm{eV} / \mathrm{q}$. (d) Number flux of ions before deceleration is shifted by $-150 \mathrm{eV} / \mathrm{q}$. The shifted lines of (c) and (d) correspond well with the number fluxes of electrons/ions after acceleration/deceleration.

the gyro-radius of the incident solar wind ions below the altitude of Kaguya was comparable or larger than the size of the small magnetic anomalies on the lunar surface, and the variation of the magnetic field intensity along the ion trajectory was quite large, the reflection of the ions was non-adiabatic. The reason why reflected ions were observed over a much larger area than the magnetic anomaly region can be explained by the non-adiabatic scattering motion of the reflected ions. Although the decelerated incident so- lar wind ions were slightly heated, the ions reflected back at the lower altitude were much more heated. Some heating mechanism of ions should exist at lower altitude than Kaguya. Figure 7 schematically summarizes the measured plasma features over a magnetic anomaly.

The electron acceleration and ion deceleration over the magnetic anomalies were observed for almost all the encounters with magnetic anomalies at $\sim 25 \mathrm{~km}$ altitude. We have investigated the acceleration energy of the electrons 
(a) Density $\left(/ \mathrm{cm}^{3}\right)$

\section{Kaguya MAP-PACE VELOCITY MOMENT}

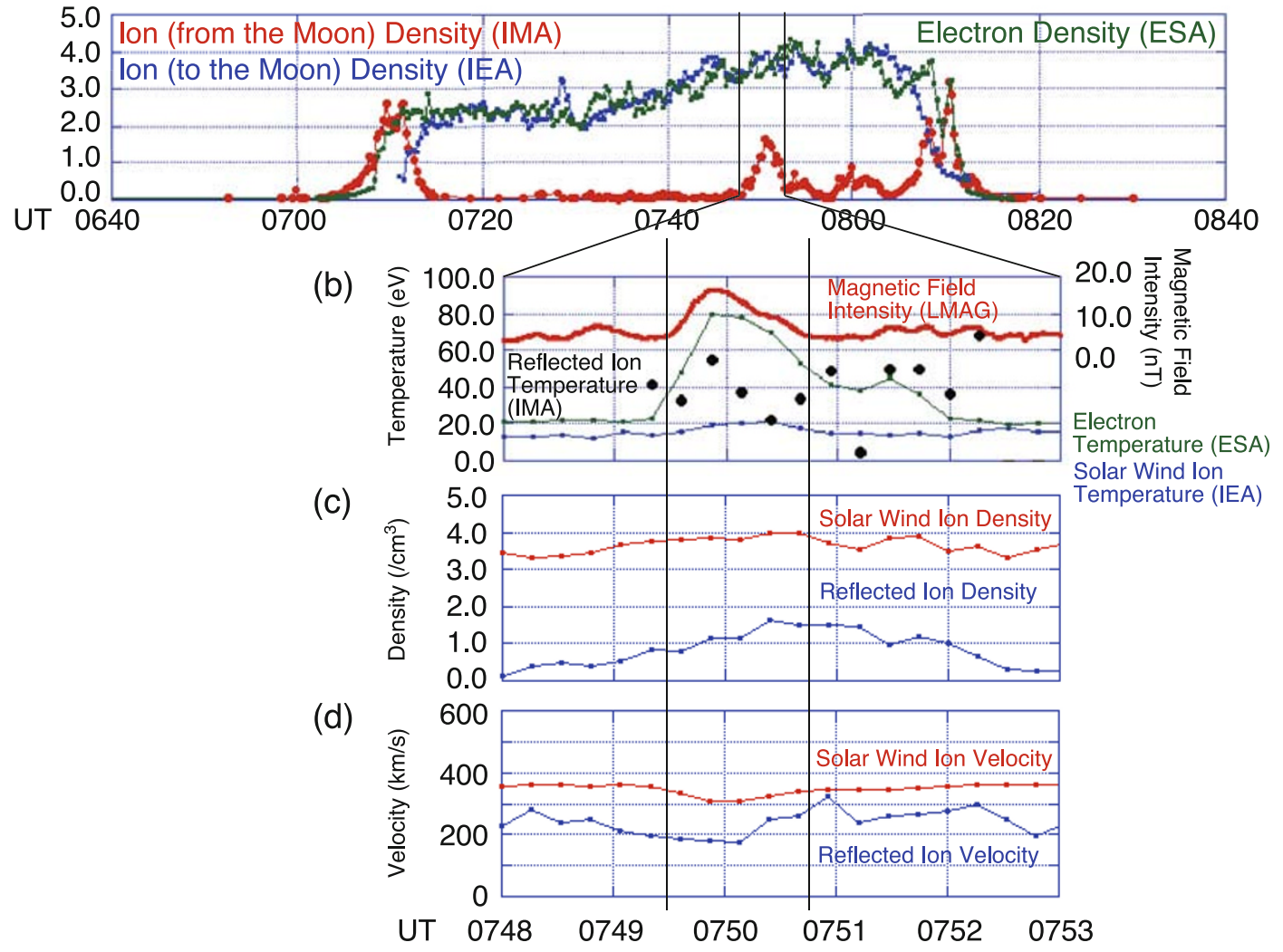

Fig. 6. Velocity moments during the same time interval as in Fig. 3. (a) Density of the ions from the Moon observed by IMA, density of the ions to the Moon observed by IEA and density of the electrons observed by ESA. (b) Magnetic field intensity, electron temperature observed by ESA, solar wind ion temperature observed by IEA and reflected ion temperature observed by IMA. (c) Solar wind ion density and reflected ion density. (d) Solar wind ion velocity and reflected ion velocity.

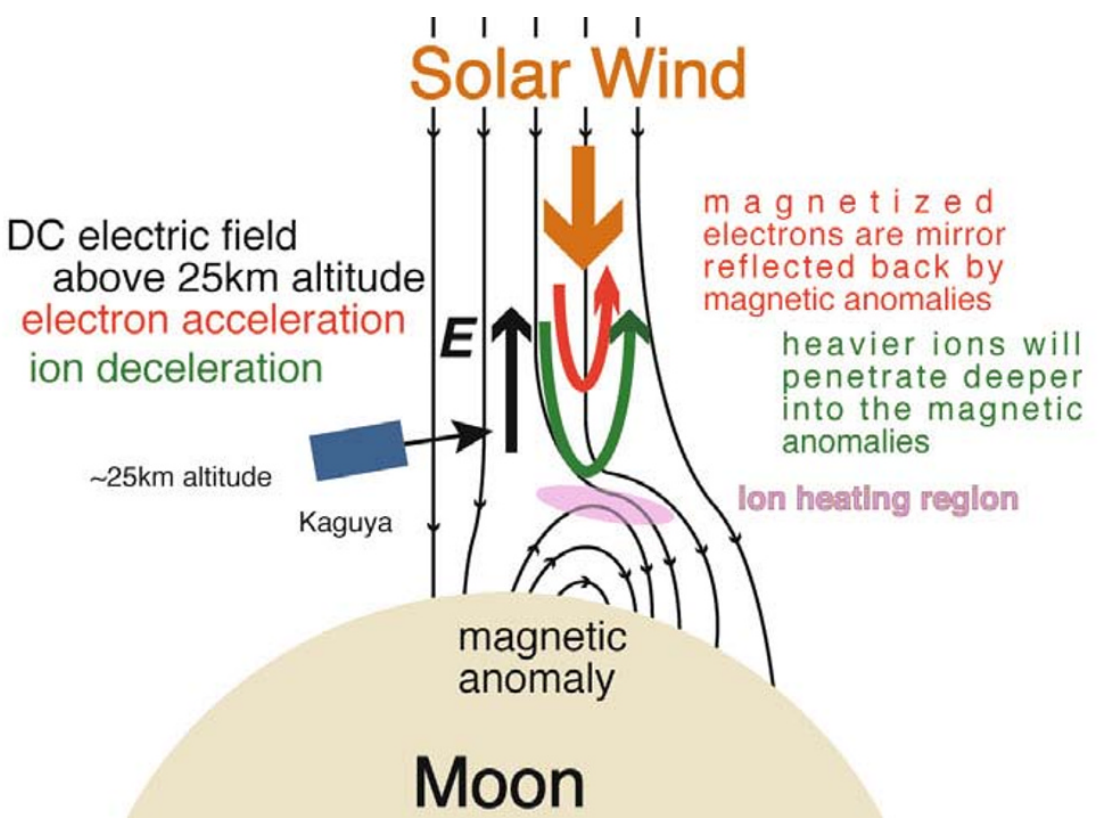

Fig. 7. A schematic figure showing the plasma structure over a magnetic anomaly.

and the deceleration energy of the ions for 13 examples obtained on 23 April 2009 when the perilune altitude was $25 \mathrm{~km}$ over the South Pole Aitken region on the dayside of the Moon. Figure 8 shows the result. The acceleration energy of the electrons and the deceleration energy of the ions have a positive correlation. Assuming that the electron acceleration $\left(E_{\text {eacc }}\right)$ and ion deceleration $\left(E_{\text {idec }}\right)$ are proportional to each other, we have obtained the relation 
Solar Wind Proton Deceleration

/ Electron Acceleration

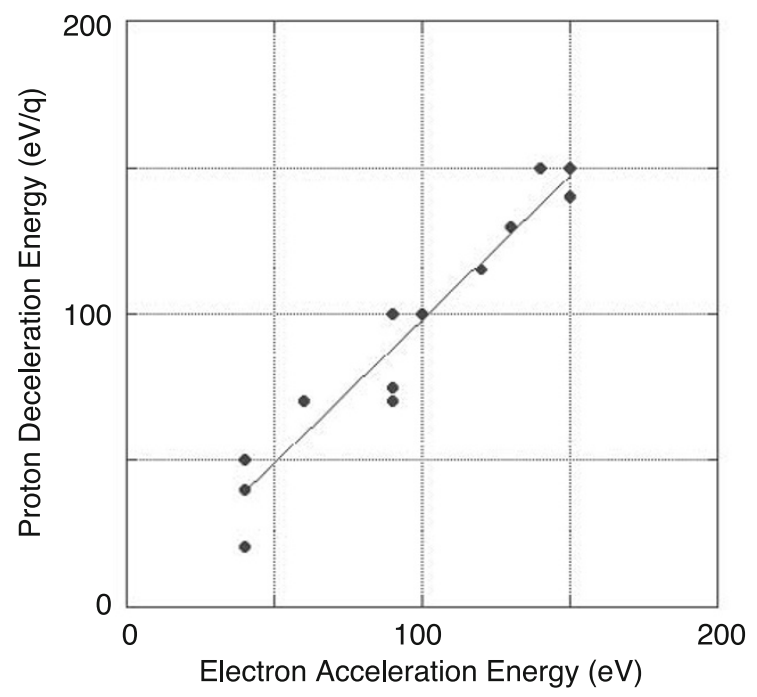

Fig. 8. Relation between acceleration energy of electrons $\left(E_{\text {eacc }}\right)$ and deceleration energy of protons $\left(E_{\text {idec }}\right)$ over magnetic anomalies. Thirteen examples were obtained on 23 April 2009 when the perilune altitude of Kaguya was $25 \mathrm{~km}$ over the South Pole Aitken region on the dayside of the Moon. The regression line on the figure is $E_{\text {idec }}=0.98 E_{\text {eacc }}$. The correlation coefficient is 0.965 .

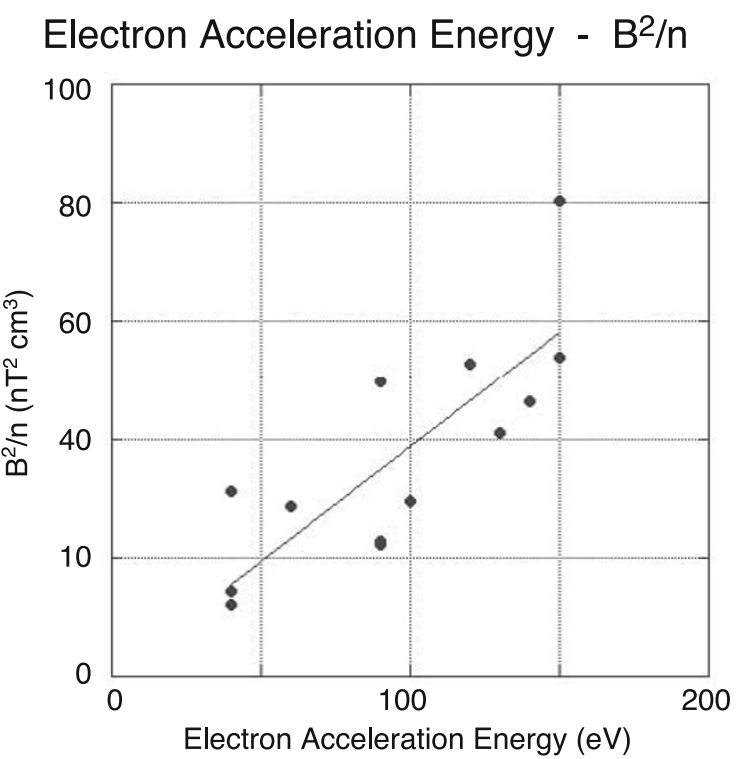

Fig. 9. Relation between electron acceleration energy and $B^{2} / n$ for the same 13 examples shown in Fig. 8 . There is a correlation coefficient of 0.798 between the electron acceleration energy and $B^{2} / n$.

$E_{\text {idec }}=0.98 E_{\text {eacc }}$ with a high correlation coefficient of 0.965 . Therefore we can say that the electron acceleration energy and the ion deceleration energy correspond with each other.

The size of the lunar magnetic anomaly shown in Fig. 4 over which the electron acceleration and ion deceleration were observed was comparable to or smaller than the ion inertial length. The size of the magnetic anomaly can be calculated as $\sim 100 \mathrm{~km}$ by multiplying the orbital speed of $\sim 1.6 \mathrm{~km} / \mathrm{s}$ and the detected period of $\sim 60 \mathrm{~s}$ while the ion inertial length was $\sim 115 \mathrm{~km}$ since the ion density was $\sim 4 \mathrm{~cm}^{-3}$. If the electric field over the magnetic anomaly is generated by the decoupling of the motions of the ions and electrons on ion inertial length scales, Hall-MHD is appli- cable and the following relation should be satisfied. Assuming infinite plasma conductivity, the generalized Ohm's law can be written as

$$
\begin{aligned}
\mathbf{E} & =-\mathbf{V}_{\mathrm{i}} \times \mathbf{B}+\frac{1}{n_{\mathrm{e}} e} \mathbf{J} \times \mathbf{B}-\frac{1}{n_{\mathrm{e}} e} \nabla P_{\mathrm{e}} \\
& =-\mathbf{V}_{\mathrm{i}} \times \mathbf{B}+\frac{1}{\mu_{0} n_{\mathrm{e}} e}(\nabla \times \mathbf{B}) \times \mathbf{B}-\frac{1}{n_{\mathrm{e}} e} \nabla P_{\mathrm{e}}
\end{aligned}
$$

where $\mathbf{E}$ is the electric field vector, $\mathbf{V}_{\mathrm{i}}$ is the ion velocity vector, $\mathbf{B}$ is the magnetic field vector, $\mathbf{J}$ is the current density vector, $P_{\mathrm{e}}$ is the electron pressure, $n_{\mathrm{e}}$ is the electron density, $e$ is the elementary charge, and $\mu_{0}$ is the vacuum magnetic permeability. When the magnetic field is piled-up as the solar wind approaches the magnetic anomaly on the lunar 
surface (in other words, when the solar wind is not parallel to IMF), the Hall term in $(2): \frac{1}{\mu_{0} n_{\mathrm{e}} e}(\nabla \times \mathbf{B}) \times \mathbf{B}$ is the antimoonward electric field over the magnetic anomaly. Therefore $E_{n} \propto B^{2} / n L$, where $E_{n}$ is the anti-moonward electric field intensity, $B$ is the magnetic field, $n$ is the density, and $L$ is the scale size of the magnetic anomaly. Since $L$ is the scale size of the magnetic anomaly, $E_{n} L$ corresponds to the potential drop over the magnetic anomaly. Figure 9 shows the relation between the electron acceleration energy and $B^{2} / n$. The peak magnetic field intensity observed by LMAG is used for $B$ while the solar wind plasma density observed by PACE is used for $n$. Although the data points scatter, there exists a positive correlation between the electron acceleration energy and $B^{2} / n$ with a correlation coefficient of 0.798 . Since there exists a positive correlation between $E_{n} L$ and $B^{2} / n$, as predicted by Hall-MHD, the electric field over the magnetic anomaly is consistent with the electric field generated by the decoupling of the motions of the ions and the electrons on ion inertial length scales. While the ions are mostly decelerated by the perpendicular electric field, the electrons are accelerated by the associated parallel electric field. In the one-dimensional picture (electric field normal to the surface that varies along the altitude) developed here, the electric field is electrostatic and the potential drop along a field line should agree with that along the solar wind direction. In the traditional framework denoted by (2), the parallel $E$-field is sustained by the electron pressure gradient. Some velocity-space-relaxation processes are needed to make the assumption fully valid. The lack of such processes makes the beam features remain in the electron distribution function (see Fig. 4) and enables us to infer the origin of the electric field structure.

Here one should note that the discussion here applies to the origin of the electric field above the Kaguya altitude where the bulk flow deceleration of the solar wind and the associated piling-up of the magnetic field is the dominating process. Closer to the lunar surface, the particle nature of the ions and electrons will be more important for understanding the plasma structure around the magnetic anomalies.

When the solar wind plasma collides with a lunar magnetic anomaly, the magnetic anomaly is compressed by the solar wind plasma and the solar wind will impact the lunar surface if the magnetic energy of the magnetic anomaly is lower than the energy of the solar wind plasma bulk motion. In case of the solar wind plasma shown in Fig. 6, the intensity of the magnetic field that can stand off the solar wind plasma bulk motion (with bulk velocity of $350 \mathrm{~km} / \mathrm{s}$ and density of $4.0 \mathrm{~cm}^{-3}$ ) is calculated as $45 \mathrm{nT}$. The measured magnetic field intensity at Kaguya was $16 \mathrm{nT}$. It is possible that the magnetic field between Kaguya and the lunar surface is stronger than $45 \mathrm{nT}$ and the solar wind plasmas are reflected back, though this depends on the location and structure of the source of the magnetic anomaly. In addition to the reflection by magnetic field, the anti-moonward electric field over magnetic anomalies will assist ion reflection.

Although the plasma structure over lunar magnetic anomalies has become clear due to this simultaneous observation of low energy electrons and ions, it has turned out that there exists an additional interaction region where the incident ions are significantly heated at a lower altitude than Kaguya at $\sim 25 \mathrm{~km}$ altitude. In order to fully understand the MAP-PACE observations over magnetic anomalies, future hybrid or full particle simulations are indispensable.

Acknowledgments. The authors wish to express their sincere thanks to all the team members of MAP for their great support in processing and analyzing the MAP data. The authors thank the ACE MAG instrument team and the ACE Science Center for providing the ACE data. This work was supported by JSPS Grantin-Aid for Scientific Research (B) 21340143.

Guest editor M. Yamauchi thanks E. Harnett and an anonymous reviewer in evaluating this paper.

\section{References}

Anderson, K., R. Lin, R. McGuire, and J. McCoy, Measurement of lunar and planetary magnetic fields by reflection of low energy electrons, Space Sci. Insr., 1, 439-470, 1975.

Colburn, D., J. Mihalov, and C. Sonett, Magnetic observations of the lunar cavity, J. Geophys. Res., 76, 2940-2957, 1971.

Criswell, D., Photoelectrons and solar wind/lunar limb interaction, The Moon, 7, 202-238, 1973.

Fuller, M., Lunar magnetism, Rev. Geophys. Space Phys., 12, 23-69, 1974. Halekas, J., D. Brain, D. Mitchell, R. Lin, and L. Harrison, On the occurrence of magnetic enhancements caused by solar wind interaction with lunar crustal fields, Geophys. Res. Lett., 33, L08106, 2006.

Halekas, J., D. Brain, R. Lin, and D. Mitchell, Solar wind interaction with lunar crustal magnetic anomalies, Adv. Space Res., 41, 1319-1324, 2008a.

Halekas, J., G. Delory, D. Brain, R. Lin, and D. Mitchell, Density cavity observed over a strong lunar crustal magnetic anomaly in the solar wind: A mini-magnetosphere?, Planet. Space Sci., 56, 941-946, 2008b.

Halekas, J., Y. Saito, G. Delory, and W. Farrell, New views of the lunar plasma environment, Planet. Space Sci., doi:10.1016/j.pss.2010.08.011, 2010 (in press).

Harnett, E. and R. Winglee, Two dimensional MHD simulations of the solar wind interaction with magnetic field anomalies on the surface of the Moon, J. Geophys. Res., 105, 24,997-25,007, 2000.

Harnett, E. and R. Winglee, 2.5D Particle and MHD simulations of minimagnetospheres at the Moon, J. Geophys. Res., 107, 1421, 2002.

Harnett, E. and R. Winglee, 2.5-D fluid simulations of the solar wind interacting with multiple dipoles on the surface of the Moon, J. Geophys. Res., 108, 1088, 2003.

Howe, H., R. Lin, R. McGuire, and K. Anderson, Energetic electron scattering from the lunar remanent magnetic field, Geophys. Res. Lett., 1, 101-104, 1974.

Kato, M., S. Sasaki, Y. Takizawa, and the Kaguya project team, The Kaguya mission overview, Space Sci. Rev., 154, 3-19, 2010.

Kurata, M., H. Tsunakawa, Y. Saito, H. Shibuya, M. Matsushima, and H. Shimizu, Mini-magnetosphere over the Reiner Gamma magnetic anomaly region on the Moon, Geophys. Res. Lett., 32, L24205, 2005.

Lin, R., D. Mitchell, D. Curtis, K. Anderson, C. Carlson, J. McFadden, M. Acuna, L. Hood, and A. Binder, Lunar surface magnetic fields and their interaction with the solar wind: results from Lunar Prospector, Science, 281, 1480-1484, 1998.

Lue, C., Y. Futaana, S. Barabash, M. Wieser, M. Holmström, A. Bhardwaj, M. Dhanya, and P. Wurz, Strong influence of lunar crustal fields on the solar wind flow, Geophys. Res. Lett., 38, L03202, 2011.

Matsushima, M., H. Tsunakawa, Y. Iijima, S. Nakazawa, A. Matsuoka, S. Ikegami, T. Ishikawa, H. Shibuya, H. Shimizu, and F. Takahashi, Magnetic cleanliness program under control of electromagnetic compatibility for the SELENE (Kaguya) spacecraft, Space Sci. Rev., 154, 253-264, 2010.

Ness, N., K. Behannon, H. Taylor, and Y. Whang, Perturbations of the interplanetary magnetic field by the lunar wake, J. Geophys. Res., 73, 3421-3440, 1968.

Russell, C. and B. Lichtenstein, On the source of lunar limb compressions, J. Geophys. Res., 80, 4700-4711, 1975.

Saito, Y., S. Yokota, K. Asamura, T. Tanaka, R. Akiba, M. Fujimoto, H. Hasegawa, H. Hayakawa, M. Hirahara, M. Hoshino, S. Machida, T. Mukai, T. Nagai, T. Nagatsuma, M. Nakamura, K. Oyama, E. Sagawa, S. Sasaki, K. Seki, and T. Terasawa, Low energy charged particle measurement by MAP-PACE onboard SELENE, Earth Planets Space, 60, 
375-385, 2008a.

Saito, Y., S. Yokota, T. Tanaka, K. Asamura, M. Nishino, M. Fujimoto, H. Tsunakawa, H. Shibuya, M. Matsushima, H. Shimizu, F. Takahashi, T. Mukai, and T. Terasawa, Solar wind proton reflection at the lunar surface: Low energy ion measurement by MAP-PACE onboard SELENE (KAGUYA), Geophys. Res. Lett., 35, L24205, 2008 b.

Saito, Y., S. Yokota, K. Asamura, T. Tanaka, M. Nishino, T. Yamamoto, Y. Terakawa, M. Fujimoto, H. Hasegawa, H. Hayakawa, M. Hirahara, M. Hoshino, S. Machida, T. Mukai, T. Nagai, T. Nagatsuma, T. Nakagawa, M. Nakamura, K. Oyama, E. Sagawa, S. Sasaki, K. Seki, I. Shinohara, T. Terasawa, H. Tsunakawa, H. Shibuya, M. Matsushima, H. Shimizu, and F. Takahashi, In-flight performance and initial results of Plasma energy Angle and Composition Experiment (PACE) on SELENE (Kaguya), Space Sci. Rev., 154, 265-303, 2010.

Sharp, L., P. Coleman, Jr., B. Lichtenstein, C. Russell, and G. Schubert, Orbital mapping of the lunar magnetic field, The Moon, 7, 322-341, 1973.

Shimizu, H., F. Takahashi, N. Horii, A. Matsuoka, M. Matsushima, H. Shibuya, and H. Tsunakawa, Ground calibration of the high- sensitivity SELENE lunar magnetometer LMAG, Earth Planets Space, 60, 353$363,2008$.

Takahashi, F., H. Shimizu, M. Matsushima, H. Shibuya, A. Matsuoka, S. Nakazawa, Y. Iijima, H. Otake, and H. Tsunakawa, In-orbit calibration of the lunar magnetometer onboard SELENE (KAGUYA), Earth Planets Space, 61, 1269-1274, 2009.

Tanaka, T., Y. Saito, S. Yokota, K. Asamura, M. Nishino, H. Tsunakawa,
H. Shibuya, M. Matsushima, H. Shimizu, F. Takahashi, M. Fujimoto, T. Mukai, and T. Terasawa, First in situ observation of the Moonoriginating ions in the Earth's Magnetosphere by MAP-PACE on SELENE (KAGUYA), Geophys. Res. Lett., 36, L22106, 2009.

Tsunakawa, H., H. Shibuya, F. Takahashi, H. Shimizu, M. Matsushima, A Matsuoka, S. Nakazawa, H. Otake, and Y. Iijima, Lunar magnetic field observation and initial global mapping of lunar magnetic anomalies by MAP-LMAG onboard SELENE (Kaguya), Space Sci. Rev., 154, 219 251, 2010.

Wieser, M., S. Barabash, Y. Futaana, M. Holmström, A. Bhardwaj, R. Sridharan, M. Dhanya, A. Schaufelberger, P. Wurz, and K. Asamura, First observation of a mini-magnetosphere above a lunar magnetic anomaly using energetic neutral atoms, Geophys. Res. Lett., 37, L05103, 2010.

Yokota, S., Y. Saito, K. Asamura, and T. Mukai, Development of an ion energy mass spectrometer for application on board three-axis stabilized spacecraft, Rev. Sci. Instrum., 76, 014501-1-014501-8, 2005.

Yokota, S., Y. Saito, K. Asamura, T. Tanaka, M. Nishino, H. Tsunakawa, H. Shibuya, M. Matsushima, H. Shimizu, F. Takahashi, M. Fujimoto, T. Mukai, and T. Terasawa, First direct detection of ions originating from the Moon by MAP-PACE IMA onboard SELENE (KAGUYA), Geophys. Res. Lett., 36, L11201, 2009.

Y. Saito (e-mail: saito@stp.isas.jaxa.jp), M. N. Nishino, M. Fujimoto, T. Yamamoto, S. Yokota, H. Tsunakawa, H. Shibuya, M. Matsushima, H. Shimizu, and F. Takahashi 